\title{
SHORT syndrome
}

INSERM

\section{Source}

INSERM. (1999). Orphanet: an online rare disease and orphan drug data base. SHORT syndrome. ORPHA:3163

SHORT syndrome is a rare inherited condition of multiple anomalies whose name stands for short stature, hyperextensibility of joints, ocular depression, Rieger anomaly (see this term) and teething delay which, along with mild intrauterine growth restriction, partial lipodystrophy, delayed bone age, hernias and progeroid appearance, are manifestations of the disease. 